\title{
Vacuum fluctuations in the presence of nonlinear boundary conditions
}

\author{
C. D. Fosco ${ }^{1}$ and L. E. Oxman ${ }^{2}$ \\ ${ }^{1}$ Centro Atómico Bariloche and Instituto Balseiro, Comisión Nacional de Energía Atómica, \\ R8402AGP Bariloche, Argentina \\ ${ }^{2}$ Instituto de Física, Universidade Federal Fluminense, \\ Campus da Praia Vermelha, 24210-340 Niterói, RJ, Brazil \\ (Received 20 May 2015; published 21 December 2015)
}

\begin{abstract}
We consider a system consisting of a quantum, massless, real scalar field, in the presence of nonlinear mirrors: infinite parallel planes, upon which the field satisfies nonlinear boundary conditions. These may appear, for example, in metamaterials having nonlinear response functions. The boundary conditions are implemented by nonquadratic interaction vertices, strictly localized on the mirrors. By using the appropriate perturbative expansions, we obtain approximate expressions for the Casimir energy corresponding to weak coupling, regarding the strength of the interaction terms. We also comment on an alternative expansion scheme that may be useful when the weak coupling expansion is not justified.

DOI: 10.1103/PhysRevD.92.125021

PACS numbers: 12.20.Ds, 03.70.+k, 11.10.-z
\end{abstract}

\section{INTRODUCTION}

Quantum vacuum fluctuations may manifest themselves, under the proper circumstances, in the form of observable, macroscopic physical effects. For instance, the existence of boundary conditions for a quantum field on a nontrivial geometry can produce a Casimir force, an effect which has been evaluated for different kinds of vacuum fields, geometries, and boundary conditions [1]. Among the many important developments that have emerged in those areas, a topic which has recently received much attention has been the use of a more accurate description of the "mirrors," in other words, of the geometry and nature of the boundary conditions.

A refined description may include corrections that are an attempt to represent, for example, a departure from the idealized situation of exactly conducting, zero-width, smooth-shape mirrors. Examples of those corrections are roughness, finite temperature, and conductivity, as well as a finite width.

Leaving aside the case of nonlocal boundary interactions [2-4], for which extensive studies have been carried out $[5,6]$, imperfect boundary conditions are usually represented-at least in some scalar field models-by the introduction of a "space-dependent mass term," such that the (otherwise massless) scalar field becomes massive just at the loci of the mirrors [7], therefore "penalizing" the development of nonzero field values on the mirror. Dirichlet boundary conditions appear, in this context, wherever that space-dependent mass tends to infinity [8].

In this kind of model, the relevant properties of the mirrors correspond to a linear response approximation. A "microscopic" way to interpret this approximation, in terms of quantum field theory, amounts to using a truncated version of the expansion of the effective action, for the vacuum field, due to the microscopic degrees of freedom living on the mirrors, to the second order in the vacuum field $[9,10]$.
On the other hand, neglecting higher-order terms in the expansion can be expected to be a reliable approximation whenever the magnitude of the quadratic term is large in comparison with the subleading (usually quartic) one. Indeed, since a large quadratic term penalizes the existence of a nonzero field configuration around the corresponding mirror, the contributions from higher-order terms (since they involve higher powers of the field) are likely to be suppressed. It is the main purpose of this paper to study the consequences on the Casimir energy due to the presence of that kind of nonlinearity, having in mind cases where the conditions to discard it are not necessarily met. We note that the inclusion of nonlinearities in the study of boundary effects in classical electrodynamics has a long-standing tradition [11], since it is certainly relevant to situations where high field intensities are involved. That is not the situation with the Casimir effect, where the motivation to include nonlinearities, even at small intensities, can appear when considering some nonlinear metamaterials [12]. For calculations in systems with nonlinearities in the bulk, and standard boundary conditions, see Refs. [13,14].

This paper is organized as follows. In Sec. II we define the model and its relevant properties, and in Sec. III we consider its vacuum energy within the context of the functional integral formalism. Then, in Sec. IV we present a study of the weakcoupling expansion for the nonlinearities. In Sec. V we consider a weak-coupling expansion adapted to the case when boundary conditions have both linear and nonlinear parts. An alternative, complementary expansion is introduced and considered in Sec. VI; it corresponds to a situation where there is a small correction on top of a Dirichlet boundary condition. Finally, in Sec. VII we present our conclusions.

\section{THE MODEL}

The model that we consider involves a real massless scalar vacuum field $\varphi(x)$ in $3+1$ dimensions, coupled to 
two flat, parallel, zero-width mirrors, denoted by $L$ and $R$, which occupy the planes $x_{3}=0$ and $x_{3}=a$, respectively. Euclidean coordinates shall be denoted by $x \equiv\left(x_{0}, x_{1}, x_{2}, x_{3}\right)$, and we will also use a specific notation $x_{\|} \equiv\left(x_{0}, x_{1}, x_{2}\right)$ for the coordinates which are parallel to the mirrors' planes (on which we assume the existence of translation invariance). Alongside the last convention, we shall use letters from the beginning of the greek alphabet $(\alpha, \beta, \ldots)$ to denote indices which run over the values 0,1 , and 2 .

The media inside each mirror is assumed to be strictly confined to the respective plane, so that the full Euclidean action for the system, $\mathcal{S}(\varphi)$, naturally decomposes as follows:

$$
\mathcal{S}(\varphi)=\mathcal{S}_{f}(\varphi)+\mathcal{S}_{I}(\varphi),
$$

where $\mathcal{S}_{f}$ denotes the free action (i.e., in the absence of mirrors) for the real scalar field,

$$
\mathcal{S}_{f}(\varphi)=\frac{1}{2} \int_{x}(\partial \varphi)^{2},
$$

and

$$
\mathcal{S}_{I}(\varphi)=\mathcal{S}_{L}(\varphi)+\mathcal{S}_{R}(\varphi)
$$

while $\mathcal{S}_{L}$ and $\mathcal{S}_{R}$ account for the coupling between $\varphi$ and the respective mirror. These terms shall be assumed to have a similar structure. We will endow them with, for the sake of simplicity, a local form, confined to a $2+1$-dimensional spacetime, with the world volumes generated by the static mirrors during the course of (trivial) time evolution:

$$
\mathcal{S}_{L}=\int_{x_{\|}} \mathcal{V}_{L}\left[\varphi\left(x_{\|}, 0\right)\right], \quad \mathcal{S}_{R}=\int_{x_{\|}} \mathcal{V}_{R}\left[\varphi\left(x_{\|}, a\right)\right],
$$

where $\mathcal{V}_{L}$ and $\mathcal{V}_{R}$ are local functions of their arguments, involving no derivatives of the fields.

From the classical equations of motion that follow from the real-time version of the action for $\varphi$, we see that they imply the boundary conditions

$$
\left\{\begin{array}{l}
\partial_{3} \varphi\left(x_{\|}, 0_{+}\right)-\partial_{3} \varphi\left(x_{\|}, 0_{-}\right)=\frac{\partial \mathcal{V}_{L}}{\partial \varphi}\left[\varphi\left(x_{\|}, 0\right)\right], \\
\partial_{3} \varphi\left(x_{\|}, a_{+}\right)-\partial_{3} \varphi\left(x_{\|}, a_{-}\right)=\frac{\partial \mathcal{V}_{R}}{\partial \varphi}\left[\varphi\left(x_{\|}, a\right)\right]
\end{array}\right.
$$

which necessarily introduce nonlinearities as soon as one of the functions $\mathcal{V}_{L}$ or $\mathcal{V}_{R}$ involves more than two powers of its argument. A consequence of this very same property is the following: when there are more than two powers of the field in one of the mirrors, the quantum equations of motion for $\varphi$-namely, the equations for its mean value $\langle\varphi\rangle$-are different to their classical counterparts. Indeed, from $0=\int \mathcal{D} \varphi \frac{\delta}{\delta \varphi(x)} e^{-\mathcal{S}(\varphi)}$, we obtain

$$
\begin{gathered}
\square\langle\varphi(x)\rangle+\delta\left(x_{3}\right)\left\langle\frac{\partial \mathcal{V}_{L}}{\partial \varphi}\left[\varphi\left(x_{\|}, 0\right)\right]\right\rangle \\
+\delta\left(x_{3}-a\right)\left\langle\frac{\partial \mathcal{V}_{R}}{\partial \varphi}\left[\varphi\left(x_{\|}, a\right)\right]\right\rangle,
\end{gathered}
$$

from which linear boundary conditions are obtained only when the potentials $\mathcal{V}_{L, R}$ are quadratic. On the contrary, for nonquadratic interactions the equation involves a Green's function with more than one field, and the resulting system of equations does not close; in other words, it becomes infinite.

\section{VACUUM ENERGY}

The vacuum energy $E$ will be obtained from the effective action $\Gamma$ (for the static configuration of two parallel planes already defined) when evaluated for a long, yet finite, time interval of length $T$,

$$
E=\lim _{T \rightarrow \infty} \frac{\Gamma}{T},
$$

where $\Gamma=-\log \mathcal{Z}$, and $\mathcal{Z}$ denotes the Euclidean vacuum transition amplitude,

$$
\mathcal{Z}=\int \mathcal{D} \varphi e^{-\mathcal{S}(\varphi)}
$$

and the action is evaluated on the (Euclidean) time interval $\left[-\frac{T}{2}, \frac{T}{2}\right]$.

By factoring out the partition function corresponding to $\mathcal{S}_{f}$, we see that $\mathcal{Z}$ may be rewritten in the equivalent way

$$
\mathcal{Z}=\mathcal{Z}_{f} \times \mathcal{Z}_{I}
$$

with

$$
\mathcal{Z}_{f}=\int \mathcal{D} \varphi e^{-\mathcal{S}_{f}(\varphi)}
$$

and

$$
\mathcal{Z}_{I}=\left\langle e^{-\mathcal{S}_{I}(\varphi)}\right\rangle
$$

where we have introduced a "Gaussian average" symbol $\langle\ldots\rangle$ to denote the functional averaging with the weight defined by $\mathcal{S}_{f}$; namely, for any expression, its average is given by

$$
\langle\ldots\rangle \equiv \frac{\int \mathcal{D} \varphi \ldots e^{-\mathcal{S}_{f}(\varphi)}}{\int \mathcal{D} \varphi e^{-\mathcal{S}_{f}(\varphi)}}
$$

Then,

$$
\Gamma=\Gamma_{f}+\Gamma_{I}
$$


where $\Gamma_{f}=-\log \mathcal{Z}_{f}$ and $\Gamma_{I}=-\log \mathcal{Z}_{I}$. Note that $\Gamma_{f}$ yields the vacuum energy corresponding to the free field system, while $\Gamma_{I}$ contains contributions due to the presence of the boundary conditions.

Since the Casimir force is insensitive to $\Gamma_{f}$, we shall discard that contribution in what follows. Besides, note that $\Gamma, \Gamma_{f}$, and $\Gamma_{I}$ are not only proportional to $T$, but also to $L^{2}$, the area of the mirrors. This is just a manifestation of the fact that the system has translation invariance along the two parallel directions to the mirrors, as well as being time independent. Thus rather than working with energies, which are extensive and therefore proportional to $L^{2}$, we will use instead energies per unit area, denoted by $\mathcal{E}, \mathcal{E}_{f}$, and $\mathcal{E}_{I}$, respectively. Thus, the interesting quantity shall be

$$
\mathcal{E}_{I}=-\lim _{T, L \rightarrow \infty}\left[\frac{1}{T L^{2}} \log \left\langle e^{-\mathcal{S}_{I}(\varphi)}\right\rangle\right] .
$$

Since we just need to keep terms that do contribute to the Casimir force, we can also subtract from $E_{I}$ contributions which - although sensitive to the existence of the boundary conditions-are independent of the distance $a$ between the mirrors. That is the case of the mirrors' self-energies which - although they certainly may depend on the details of each interaction term $\mathcal{S}_{L}$ and $\mathcal{S}_{R}$-are independent of the distance $a$ between $L$ and $R$.

\section{WEAK-COUPLING EXPANSION}

Let us calculate here the contribution to the Casimir energy due to purely nonlinear coupling terms, under the assumption that those terms are small. The procedure is entirely analogous, although applied to a nonlinear medium, to the approach followed, for example, in Refs. [15] and [16] to derive exact results in the weak-coupling regime of the static Casimir effect.

The perturbative expansion, taking as zeroth order the free action $\mathcal{S}_{f}$, amounts to expanding $\Gamma_{I}$ in powers of $\mathcal{S}_{I}$,

$$
\Gamma_{I}=\Gamma_{I}^{(1)}+\Gamma_{I}^{(2)}+\ldots
$$

where the superscript denotes the order (in $\mathcal{S}_{I}$ ) of each term in the perturbative expansion.

Up to the second order, the explicit form of the terms is as follows:

$$
\Gamma_{I}^{(1)}=\left\langle\mathcal{S}_{I}\right\rangle,
$$

and

$$
\Gamma_{I}^{(2)}=-\frac{1}{2}\left[\left\langle\left(\mathcal{S}_{I}\right)^{2}\right\rangle-\left\langle\mathcal{S}_{I}\right\rangle^{2}\right]
$$

Regarding the first-order term, we note that, since the quantum averaging is defined with the free action, the result is a sum of two self-energy terms, each one independent of the distance $a$ between the two mirrors. Thus, there is no contribution from this term to the Casimir interaction energy.

Let us now consider the second-order term for the concrete example of mirrors described by the terms

$$
\begin{aligned}
\mathcal{S}_{L} & =\int_{x_{\|}} \frac{g_{L}}{k_{L} !}:\left[\varphi\left(x_{\|}, 0\right)\right]^{k_{L}}:, \\
\mathcal{S}_{R} & =\int_{x_{\|}} \frac{g_{R}}{k_{R} !}:\left[\varphi\left(x_{\|}, a\right)\right]^{k_{R}}:,
\end{aligned}
$$

where the normal-order symbol means that contractions at the same vertex are to be discarded. ${ }^{1}$

The second-order term can then be written as follows:

$$
\Gamma_{I}^{(2)}=-\left\langle\mathcal{S}_{L} \mathcal{S}_{R}\right\rangle
$$

More explicitly, one can see that $\Gamma_{I}^{(2)}$ vanishes unless $k_{L}=k_{R} \equiv k$, and

$$
\begin{aligned}
\Gamma_{I}^{(2)} & =-\frac{g_{L} g_{R}}{k !} \int_{x_{\|}, x_{\|}^{\prime}}\left[\left\langle\varphi\left(x_{\|}, a\right) \varphi\left(x_{\|}^{\prime}, 0\right)\right\rangle\right]^{k} \\
& =-\frac{g_{L} g_{R}}{k !} T L^{2} \int_{x_{\|}}\left[\left\langle\varphi\left(x_{\|}, a\right) \varphi\left(0_{\|}, 0\right)\right\rangle\right]^{k},
\end{aligned}
$$

where

$$
\left\langle\varphi\left(x_{\|}, x_{3}\right) \varphi\left(y_{\|}, y_{3}\right)\right\rangle=\frac{1}{4 \pi^{2}\left[\left(x_{\|}-y_{\|}\right)^{2}+\left(x_{3}-y_{3}\right)^{2}\right]} .
$$

The fact that the nonvanishing contributions to the Casimir interaction appear only for $k_{L}=k_{R}$ is represented in Fig. 1, for the particular case $k_{L}=k_{R}=4$.

A rather straightforward calculation yields for the interaction energy per unit area

$$
\mathcal{E}_{I}^{(2)}=-\frac{g_{L} g_{R}}{k !} \frac{\pi^{1 / 2} \Gamma(k-3 / 2)}{2(2 \pi)^{2 k-1} \Gamma(k)} \frac{1}{a^{2 k-3}},
$$

which is an expression formally valid for any $k>3 / 2$, which is the condition to ensure convergence of the spatial integral in Eq. (20), for large values of $\left|x_{\|}\right|$. It is interesting to note that, for "perfect" boundary conditions (Dirichlet, for instance), the only dimensionful quantity which appears in the energy density is the distance $a$; this implies that $\mathcal{E}_{I} \propto \frac{1}{a^{3}}$. On the other hand, the perturbative result above should be valid for weak coupling, and one cannot therefore take the limit when the couplings tend to infinity. However,

\footnotetext{
${ }^{1}$ The normal-order symbol could be dropped at the expense of adding a certain number of counterterms, which form a polynomial of degree $k_{L, R}-2$ at the respective mirror, having the parity of the integer $k_{L, R}$.
} 


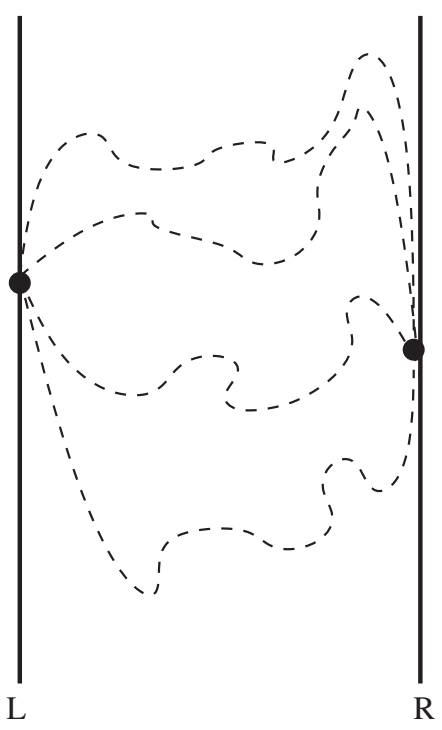

FIG. 1. The first nontrivial correction in the weak-coupling regime, for $k=4$.

we may note that, since the mass dimension of the coupling constants is $[M]^{3-K}$, when $k=3$ they become dimensionless. Thus, in that situation the dependence with $a$ is entirely analogous to the case of Dirichlet or Neumann boundary conditions, since

$$
\left.\left[\mathcal{E}_{I}^{(2)}\right]\right|_{k \rightarrow 3}=-\frac{g_{L} g_{R}}{1536 \pi^{4} a^{3}} .
$$

We note that this dependence with the distance, for the particular case $k=3$, is dictated by dimensional analysis and therefore it should be exact. In other words, it should be valid even if a nonperturbative approach were used to calculate the energy.

It is rather straightforward to check that, for the $k=3$ case, the third-order term vanishes, except for self-energy contributions.

The weak-coupling approximation can also be applied to situations where the interaction terms are not necessarily of a polynomial form. Indeed, let us assume that the functions $\mathcal{V}_{L, R}$ can be represented in terms of their Fourier transforms, $\tilde{\mathcal{V}}_{L, R}$, respectively:

$$
\begin{aligned}
& \mathcal{V}_{L}\left[\varphi\left(x_{\|}, 0\right)\right]=\int \frac{d \lambda_{L}}{2 \pi} \tilde{\mathcal{V}}_{L}\left(\lambda_{L}\right) e^{i \lambda_{L} \varphi\left(x_{\|}, 0\right)}, \\
& \mathcal{V}_{R}\left[\varphi\left(x_{\|}, a\right)\right]=\int \frac{d \lambda_{R}}{2 \pi} \tilde{\mathcal{V}}_{R}\left(\lambda_{R}\right) e^{i \lambda_{R} \varphi\left(x_{\|}, a\right)}
\end{aligned}
$$

We also assume that the constant and linear parts of the interaction terms vanish, namely, that the functions $\mathcal{V}_{L, R}$, as well as their derivatives with respect to the field, vanish at the origin (when the field equals zero):

$$
\mathcal{V}_{L}[0]=\mathcal{V}_{R}[0]=0, \quad \mathcal{V}_{L}^{\prime}[0]=\mathcal{V}_{R}^{\prime}[0]=0
$$

These conditions amount to considering only interaction terms which introduce nontrivial fluctuations.

In Fourier space, they amount to

$$
\begin{gathered}
\int \frac{d \lambda_{L}}{2 \pi} \tilde{\mathcal{V}}_{L}\left(\lambda_{L}\right)=\int \frac{d \lambda_{R}}{2 \pi} \tilde{\mathcal{V}}_{R}\left(\lambda_{L}\right)=0, \\
\int \frac{d \lambda_{L}}{2 \pi} \lambda_{L} \tilde{\mathcal{V}}_{L}\left(\lambda_{L}\right)=\int \frac{d \lambda_{R}}{2 \pi} \lambda_{R} \tilde{\mathcal{V}}_{R}\left(\lambda_{L}\right)=0 .
\end{gathered}
$$

The normal-ordered versions of the coupling terms are given by [17]

$$
\begin{aligned}
& : \mathcal{V}_{L}\left[\varphi\left(x_{\|}, 0\right)\right]:=\int \frac{d \lambda_{L}}{2 \pi} \tilde{\mathcal{V}}_{L}\left(\lambda_{L}\right): e^{i \lambda_{L} \varphi\left(x_{\|}, 0\right)}:, \\
& : \mathcal{V}_{R}\left[\varphi\left(x_{\|}, a\right)\right]:=\int \frac{d \lambda_{R}}{2 \pi} \tilde{\mathcal{V}}_{R}\left(\lambda_{L}\right): e^{i \lambda_{R} \varphi\left(x_{\|}, a\right)}:
\end{aligned}
$$

where

$$
: e^{i \lambda_{L} \varphi\left(x_{\|}, 0\right)}:=e^{i \lambda_{L} \varphi\left(x_{\|}, 0\right)+\frac{1}{2} \lambda_{L}^{2}\left\langle\varphi\left(x_{\|}, 0\right) \varphi\left(x_{\|}, 0\right)\right\rangle}
$$

and

$$
: e^{i \lambda_{R} \varphi\left(x_{\|}, a\right)}:=e^{i \lambda_{R} \varphi\left(x_{\|}, a\right)+\frac{1}{2} \lambda_{R}^{2}\left\langle\varphi\left(x_{\|}, a\right) \varphi\left(x_{\|}, a\right)\right\rangle} .
$$

Thus, the first nontrivial contribution to the interaction energy comes from

$$
\begin{aligned}
\Gamma_{I}^{(2)}= & -\int \frac{d \lambda_{L}}{2 \pi} \int \frac{d \lambda_{R}}{2 \pi} \tilde{\mathcal{V}}_{L}\left(\lambda_{L}\right) \tilde{\mathcal{V}}_{R}\left(\lambda_{R}\right) \\
& \times \int_{x_{\|}, x_{\|}^{\prime}}\left\langle: e^{i \lambda_{L} \varphi\left(x_{\|}, 0\right)}:: e^{i \lambda_{R} \varphi\left(x_{\|}^{\prime}, a\right)}:\right\rangle \\
= & -T L^{2} \int \frac{d \lambda_{L}}{2 \pi} \int \frac{d \lambda_{R}}{2 \pi} \tilde{\mathcal{V}}_{L}\left(\lambda_{L}\right) \tilde{\mathcal{V}}_{R}\left(\lambda_{R}\right) \\
& \times \int_{x_{\|}} e^{-\lambda_{L} \lambda_{R}\left\langle\varphi\left(x_{\|}, a\right) \varphi(0,0)\right\rangle},
\end{aligned}
$$

and the surface density of (interaction) energy may be put in the form

$\mathcal{E}_{I}^{(2)}=-4 \pi \int \frac{d \lambda_{L}}{2 \pi} \int \frac{d \lambda_{R}}{2 \pi} \tilde{\mathcal{V}}_{L}\left(\lambda_{L}\right) \tilde{\mathcal{V}}_{R}\left(\lambda_{R}\right) \int_{0}^{\infty} d r r^{2} e^{-\frac{\lambda_{L} \lambda_{R}}{4 \pi^{2} r^{2}+a^{2}}}$

This contribution should be reliable at large distances, where the field propagator can be made sufficiently small. Expanding the previous expression for large $a$, we obtain

$$
\mathcal{E}_{I}^{(2)} \sim \sum_{l=2}^{\infty} \frac{c_{l}}{a^{2 l-3}}
$$

with 


$$
\begin{aligned}
c_{l}= & \frac{(-1)^{l+1} \pi^{1 / 2} \Gamma(l-3 / 2)}{2^{2 l} \pi^{2 l-1} \Gamma(l) l !}\left(\int \frac{d \lambda_{L}}{2 \pi} \tilde{\mathcal{V}}_{L}\left(\lambda_{L}\right) \lambda_{L}^{l}\right) \\
& \times\left(\int \frac{d \lambda_{R}}{2 \pi} \tilde{\mathcal{V}}_{R}\left(\lambda_{R}\right) \lambda_{L}^{l}\right),
\end{aligned}
$$

which depends on the momenta of the Fourier transform of the "potentials." Those momenta determine the strength of the corresponding (negative) power of the distance in the contribution. The series starts at $l=2$, in view of the conditions (26).

As a concrete example, we consider the case of the Gaussian interaction terms:

$$
\begin{aligned}
& \mathcal{V}_{L}\left[\varphi\left(x_{\|}, 0\right)\right]=-\frac{\beta_{L}}{\sqrt{2 \pi \alpha_{L}}}\left\{e^{-\frac{1}{2 \alpha_{L}}\left[\varphi\left(x_{\|}, 0\right)\right]^{2}}-1\right\}, \\
& \mathcal{V}_{R}\left[\varphi\left(x_{\|}, a\right)\right]=-\frac{\beta_{R}}{\sqrt{2 \pi \alpha_{R}}}\left\{e^{-\frac{1}{2 \alpha_{R}}\left[\varphi\left(x_{\|}, a\right)\right]^{2}}-1\right\},
\end{aligned}
$$

where $\beta_{L, R}$ and $\alpha_{L, R}$ are positive constants. Note that these functions satisfy the conditions (25). Their Fourier transforms are

$$
\tilde{\mathcal{V}}_{L, R}\left(\lambda_{L, R}\right)=-\beta_{L, R}\left[e^{-\frac{1}{2} \alpha_{L, R}\left(\lambda_{L, R}\right)^{2}}-\sqrt{\frac{2 \pi}{\alpha_{L, R}}} \delta\left(\lambda_{L, R}\right)\right] .
$$

Inserting the previous expressions into the result for the leading term in the interaction energy, and after some algebra, we obtain

$$
\begin{aligned}
\mathcal{E}_{I}^{(2)}= & 2 \frac{\beta_{L} \beta_{R}}{\sqrt{\alpha_{L} \alpha_{R}}} a^{3} \int_{0}^{\infty} d x x^{2} \\
& \times\left\{1-\left[1-\frac{1}{(2 \pi)^{4} \alpha_{L} \alpha_{R} a^{4}\left(x^{2}+1\right)^{2}}\right]^{-1 / 2}\right\} .
\end{aligned}
$$

The integral can be shown to be well defined if the condition $(2 \pi)^{4} \alpha_{L} \alpha_{R} a^{4}>1$ is satisfied, which introduces a minimum distance (given the values of $\alpha_{L, R}$ ). More specifically, that minimum distance becomes larger when the constants $\alpha_{L, R}$ are smaller, i.e., when the interaction terms are more concentrated around zero (in field space). The long-distance expansion of Eq. (36) yields

$\mathcal{E}_{I}^{(2)}=-\frac{\beta_{L} \beta_{R}}{\left(\alpha_{L} \alpha_{R}\right)^{3 / 2}} \frac{1}{32768 \pi^{3}}\left[512 a^{-1}+\frac{3}{\pi^{4}}\left(\alpha_{L} \alpha_{R}\right)^{-1} a^{-5}+\ldots\right]$,

where the general features enunciated in Eq. (32) are verified.

\section{LINEAR PLUS NONLINEAR BOUNDARY CONDITIONS}

In this case, we consider mirrors which are described by coupling terms including both quadratic and nonquadratic pieces. The former can and will be treated here exactly, while the latter will be expanded in a perturbative expansion. For the quadratic part, we include mass-like terms for the fields at the mirrors, and, in order to evaluate $\mathcal{Z}$ (and $\Gamma$ ), we shall use a perturbative expansion in the nonlinear terms; we split the full action into its quadratic and quartic parts,

$$
\mathcal{S}(\varphi)=\mathcal{S}_{0}(\varphi)+\mathcal{S}_{I}(\varphi),
$$

where now $\mathcal{S}_{0}$ includes both the free action $\mathcal{S}_{f}$ and quadratic terms responsible for the linear coupling to the mirror:

$\mathcal{S}_{0}(\varphi)=\frac{1}{2} \int_{x}(\partial \varphi)^{2}+\int_{x_{\|}}\left[\frac{\mu_{L}}{2} \varphi^{2}\left(x_{\|}, 0\right)+\frac{\mu_{R}}{2} \varphi^{2}\left(x_{\|}, a\right)\right]$,

where $\mu_{L, R}$ are constants, and $\mathcal{S}_{I}$ is as in Eq. (3).

The known result for $\mathcal{E}_{0}$ is

$$
\mathcal{E}_{0}=\frac{1}{2} \int \frac{d^{3} k_{\|}}{(2 \pi)^{3}} \log \left[1-\frac{e^{-2\left|k_{\|}\right| a}}{\left(1+\frac{2\left|k_{\|}\right|}{\mu_{L}}\right)\left(1+\frac{2\left|k_{\|}\right|}{\mu_{R}}\right)}\right] .
$$

Again, as in the previous section, we need to evaluate functional averages of expressions involving powers of the scalar field. Since the functional weight is again a Gaussian, Wick's theorem for vacuum expectation values holds true, this time with a different elementary contraction. Therefore, the evaluation of each term requires the knowledge of $G$, the two-point correlation function for the scalar field, in the presence of the Gaussian weight:

$$
G(x ; y) \equiv\langle\varphi(x) \varphi(y)\rangle,
$$

where we keep the same symbol for the average as in the previous section, albeit the functional weight is determined by $\mathcal{S}_{0}$. The crucial difference with the previous section is that now the two-point correlation does have a dependence on the distance $a$, which makes the subtraction of selfenergies different. To that end, we first note that the exact form of $G$ may be explicitly found, and it can be written as follows:

$$
G(x ; y)=G_{f}(x ; y)-H(x ; y),
$$

where $G_{f}$ denotes the correlation function corresponding to the free field, i.e., in the absence of mirrors,

$$
G_{f}(x ; y)=\left\langle x\left|\left(-\partial^{2}\right)^{-1}\right| y\right\rangle=\int \frac{d^{4} k}{(2 \pi)^{4}} \frac{e^{i k \cdot(x-y)}}{k^{2}},
$$

while 


$$
\begin{aligned}
H(x ; y)= & \int \frac{d^{3} k_{\|}}{(2 \pi)^{3}} \frac{d p_{3}}{2 \pi} \frac{d q_{3}}{2 \pi} e^{i k_{\|} \cdot\left(x_{\|}-y_{\|}\right)} \frac{1}{\left(k_{\|}^{2}+p_{3}^{2}\right)\left(k_{\|}^{2}+q_{3}^{2}\right) D\left(k_{\|}\right)}\left\{\left(\frac{1}{\mu_{R}}+\frac{1}{2\left|k_{\|}\right|}\right) e^{i\left(p_{3} x_{3}-q_{3} y_{3}\right)}\right. \\
& \left.+\left(\frac{1}{\mu_{L}}+\frac{1}{2\left|k_{\|}\right|}\right) e^{i\left(p_{3}\left(x_{3}-a\right)+q_{3}\left(a-y_{3}\right)\right)}-\frac{e^{-\left|k_{\|}\right| a}}{2\left|k_{\|}\right|}\left[e^{i\left(p_{3} x_{3}+q_{3}\left(a-y_{3}\right)\right)}+e^{i\left(p_{3}\left(x_{3}-a\right)-q_{3} y_{3}\right)}\right]\right\},
\end{aligned}
$$

with

$$
D\left(k_{\|}\right)=\left(\frac{1}{\mu_{L}}+\frac{1}{2\left|k_{\|}\right|}\right)\left(\frac{1}{\mu_{R}}+\frac{1}{2\left|k_{\|}\right|}\right)-\frac{e^{-2\left|k_{\|}\right| a}}{4 k_{\|}^{2}} .
$$

When calculating the first-order term $\Gamma_{I}^{(1)}$, we face the divergences due to tadpole diagrams. Since we are working in the functional integral formalism, the would-be normalordering procedure of the operatorial formalism manifests itself here as the addition of two counterterms to the action: one of them is quadratic in the scalar field, while the other is field- and $a$-independent (a constant). We shall not write the latter explicitly.

For the case of quartic vertices with coefficients $g_{L}$ and $g_{R}$, at the first order one has to add to the action the counterterm action $\mathcal{S}_{c t}^{(1)}$ given by

$$
\begin{aligned}
\mathcal{S}_{c t}^{(1)}= & -\frac{g_{L}}{4} \int_{x_{\|}}\left[G\left(x_{\|}, 0 ; x_{\|}, 0\right)\right]_{a \rightarrow \infty}\left[\varphi\left(x_{\|}, 0\right)\right]^{2} \\
& -\frac{g_{R}}{4} \int_{x_{\|}}\left[G\left(x_{\|}, a ; x_{\|}, a\right)\right]_{a \rightarrow \infty}\left[\varphi\left(x_{\|}, a\right)\right]^{2},
\end{aligned}
$$

which subtracts the divergences in the self-energy $\Pi$ of the field (which would otherwise shift the constants $\mu_{L, R}$ ). Note that this addition to the action may be interpreted as imposing a "renormalization condition" when the mirrors are absent $(a \rightarrow \infty)$.

The new, two-legged vertices due to the counterterm action above have to be taken into account in the evaluation of the first-order correction to $\Gamma$, since they are of the same order. Putting together all the contributions, we see that

$$
\begin{aligned}
\Gamma_{I}^{(1)}= & \left.\left.\frac{3}{4 !} g_{L} \int_{x_{\|}}\left[G\left(x_{\|}, 0 ; x_{\|}, 0\right)\right]^{2}+\frac{3}{4 !} g_{R} \int_{x_{\|}}\left[G\left(x_{\|}, a ; x_{\|}, a\right)\right]^{2}\right\}-\frac{1}{4} g_{L} \int_{x_{\|}}\left[G\left(x_{\|}, 0 ; x_{\|}, 0\right)\right]_{a \rightarrow \infty} G\left(x_{\|}, 0 ; x_{\|}, 0\right)\right] \\
& \left.\left.+\frac{1}{4} g_{R} \int_{x_{\|}}\left[G\left(x_{\|}, a ; x_{\|}, a\right)\right]_{a \rightarrow \infty} G\left(x_{\|}, a ; x_{\|}, a\right)\right]\right\} .
\end{aligned}
$$

Note that the situation is analogous to the one in finitetemperature quantum field theory [18], where the normal ordering of a vertex is performed at zero temperature, and there is a remaining, temperature-dependent (here, $a$-dependent) contribution, which adds up to the free energy.

Adding constants (the constant counterterms) to Eq. (47), we may complete squares, obtaining

$$
\begin{aligned}
{\left[\Gamma_{I}^{(1)}\right]_{\mathrm{ren}}=} & \frac{3}{4 !} g_{L} \int_{x_{\|}}\left\{\left[G\left(x_{\|}, 0 ; x_{\|}, 0\right)\right]^{2}-2\left[G\left(x_{\|}, 0 ; x_{\|}, 0\right)\right]_{a \rightarrow \infty} G\left(x_{\|}, 0 ; x_{\|}, 0\right)+\left[G\left(x_{\|}, 0 ; x_{\|}, 0\right)\right]_{a \rightarrow \infty}^{2}\right\} \\
& +\frac{3}{4 !} g_{R} \int_{x_{\|}}\left\{\left[G\left(x_{\|}, a ; x_{\|}, a\right)\right]^{2}-2\left[G\left(x_{\|}, a ; x_{\|}, a\right)\right]_{a \rightarrow \infty} G\left(x_{\|}, a ; x_{\|}, a\right)+\left[G\left(x_{\|}, a ; x_{\|}, a\right)\right]_{a \rightarrow \infty}^{2}\right\},
\end{aligned}
$$

or

$$
\left[\Gamma_{I}^{(1)}\right]_{\mathrm{ren}}=\frac{3}{4 !} g_{L} \int_{x_{\|}}\left[G_{1}\left(x_{\|}, 0 ; x_{\|}, 0\right)\right]^{2}+\frac{3}{4 !} g_{R} \int_{x_{\|}}\left[G_{1}\left(x_{\|}, a ; x_{\|}, a\right)\right]^{2},
$$

with

$$
\begin{aligned}
& G_{1}\left(x_{\|}, 0 ; x_{\|}, 0\right)=G\left(x_{\|}, 0 ; x_{\|}, 0\right)-\left[G\left(x_{\|}, 0 ; x_{\|}, 0\right)\right]_{a \rightarrow \infty} \\
& G_{1}\left(x_{\|}, a ; x_{\|}, a\right)=G\left(x_{\|}, a ; x_{\|}, a\right)-\left[G\left(x_{\|}, a ; x_{\|}, a\right)\right]_{a \rightarrow \infty} .
\end{aligned}
$$


We see that

$$
\begin{aligned}
& G_{1}\left(x_{\|}, 0 ; x_{\|}, 0\right)=-H\left(x_{\|}, 0 ; x_{\|}, 0\right)+\left[H\left(x_{\|}, 0 ; x_{\|}, 0\right)\right]_{a \rightarrow \infty}, \\
& G_{1}\left(x_{\|}, a ; x_{\|}, a\right)=-H\left(x_{\|}, a ; x_{\|}, a\right)+\left[H\left(x_{\|}, a ; x_{\|}, a\right)\right]_{a \rightarrow \infty}
\end{aligned}
$$

since the free, $a$-dependent part of $G$ is canceled out.

Using the explicit formulas for $H$, we get

$$
\begin{aligned}
& G_{1}\left(x_{\|}, 0 ; x_{\|}, 0\right) \\
& =-\int \frac{d^{3} k_{\|}}{(2 \pi)^{3}} \frac{1}{\mu_{L}\left(1+\frac{\mu_{L}}{2 \mid k_{\|}}\right)\left[\left(1+\frac{2\left|k_{\|}\right|}{\mu_{L}}\right)\left(1+\frac{2\left|k_{\|}\right|}{\mu_{R}}\right) e^{2\left|k_{\|}\right| a}-1\right]}, \\
& G_{1}\left(x_{\|}, a ; x_{\|}, a\right) \\
& =-\int \frac{d^{3} k_{\|}}{(2 \pi)^{3}} \frac{1}{\mu_{R}\left(1+\frac{\mu_{R}}{2\left|k_{\|}\right|}\right)\left[\left(1+\frac{2\left|k_{\|}\right|}{\mu_{L}}\right)\left(1+\frac{2\left|k_{\|}\right|}{\mu_{R}}\right) e^{2\left|k_{\|}\right| a}-1\right]} .
\end{aligned}
$$

It is worth noting that most of what we have said before could also have been obtained for the case of momentumdependent coefficients $\mu_{L, R}$, (with an action which is a straightforward generalization of the one for constant coefficients). This allows us to consider a rather "economical" model, consisting of one where the mass dimensions of the coefficients for the quadratic terms are given by the momentum itself, so that we are just left with dimensionless coefficients. Namely,

$$
\mu_{L, R}\left(k_{\|}\right)=2 \zeta_{L, R}\left|k_{\|}\right|,
$$

where $\zeta_{L, R}$ are dimensionless constants.

In this case, the first-order contribution to the vacuum energy is

$$
\mathcal{E}^{(1)}=\frac{3}{4 ! a^{4}}\left(\frac{g_{L}}{\zeta_{L}^{2}\left(1+\zeta_{L}\right)^{2}}+\frac{g_{R}}{\zeta_{R}^{2}\left(1-\zeta_{R}\right)^{2}}\right)\left[I\left(\zeta_{L}, \zeta_{R}\right)\right]^{2},
$$

where

$$
I\left(\zeta_{L}, \zeta_{R}\right)=\frac{1}{4 \pi^{2}} \int d \rho \rho \frac{1}{\left(1+\frac{1}{\zeta_{L}}\right)\left(1+\frac{1}{\zeta_{R}}\right) e^{2 \rho}-1} .
$$

The absence of other dimensionful objects in this model beyond $a$ and the coupling constants means that the $a$ dependence of each term can be obtained rather straightforwardly. Indeed, if the term is of order $g_{L}^{n} g_{R}^{m}$, the dependence with $a$ is $\propto \frac{1}{a^{n+m+3}}$.

\section{ALTERNATIVE EXPANSION}

Finally, we will consider here an alternative expansion, still under the same general structure of the ones considered before, but such that the interaction terms can (by assumption) be represented in terms of the generalized Fourier transformations:

$$
\begin{aligned}
e^{-\mathcal{S}_{L}(\varphi)} & =\frac{1}{\mathcal{N}_{L}} \int \mathcal{D} \xi_{L} e^{-W_{L}\left(\xi_{L}\right)+i \int_{x_{\|}} \xi_{L}\left(x_{\|}\right) \varphi\left(x_{\|}, 0\right)}, \\
e^{-\mathcal{S}_{R}(\varphi)} & =\frac{1}{\mathcal{N}_{R}} \int \mathcal{D} \xi_{R} e^{-W_{R}\left(\xi_{R}\right)+i \int_{x_{\|}} \xi_{R}\left(x_{\|}\right) \varphi\left(x_{\|}, a\right)},
\end{aligned}
$$

where $\xi_{L}$ and $\xi_{R}$ are auxiliary fields, and $\mathcal{N}_{L, R}$ are normalization constants.

It may be thought of as a particular case of the previous section, namely, when the constants $\mu_{L}$ and $\mu_{R}$ tend to infinity, while the microscopic interactions are such that there still are nonvanishing small nonquadratic functions $W_{L, R}$.

We then insert the representations (56) into the definition (8) of $\mathcal{Z}$, and integrate out the scalar field $\varphi$, to obtain

$$
\mathcal{Z}=\frac{\mathcal{Z}_{f}}{\mathcal{N}_{L} \mathcal{N}_{R}} \int \mathcal{D} \xi_{L} \mathcal{D} \xi_{R} e^{-W_{q}\left(\xi_{L}, \xi_{R}\right)-W_{L}\left(\xi_{L}\right)-W_{R}\left(\xi_{R}\right)},
$$

where $W_{q}\left(\xi_{L}, \xi_{R}\right)$ denotes the quadratic form in the auxiliary fields,

$$
\begin{aligned}
W_{q}\left(\xi_{L}, \xi_{R}\right) & =\frac{1}{2} \int_{x_{\|}, x_{\|}^{\prime}} \xi_{A}\left(x_{\|}\right) K_{A B}\left(x_{\|}, x_{\|}^{\prime}\right) \xi_{B}\left(x_{\|}^{\prime}\right), \\
A & =L, R,
\end{aligned}
$$

where

$$
K_{A B}\left(x_{\|}, x_{\|}^{\prime}\right)=\int \frac{d^{3} k_{\|}}{(2 \pi)^{3}} e^{i k_{\|} \cdot\left(x_{\|}-x_{\|}^{\prime}\right)} \tilde{K}_{A B}\left(k_{\|}\right)
$$

with

$$
\left[\tilde{K}_{A B}\left(k_{\|}\right)\right]=\frac{1}{2\left|k_{\|}\right|}\left(\begin{array}{cc}
1 & e^{-\left|k_{\|}\right| a} \\
e^{-\left|k_{\|}\right| a} & 1
\end{array}\right) .
$$

Proceeding in an analogous manner to the one followed for the weak-coupling expansion, but with $W_{q}$ playing a role similar to $\mathcal{S}_{f}$, we see that

$$
\Gamma=\Gamma_{q}+\Gamma_{s}
$$

with

$$
e^{-\Gamma_{q}}=\int \mathcal{D} \xi_{L} \mathcal{D} \xi_{R} e^{-W_{q}\left(\xi_{L}, \xi_{R}\right)}
$$

and 


$$
\begin{aligned}
e^{-\Gamma_{s}} & =\left\langle e^{-W_{L}\left(\xi_{L}\right)-W_{R}\left(\xi_{R}\right)}\right\rangle_{q} \\
\langle\ldots\rangle_{q} & \equiv \frac{\int \mathcal{D} \xi_{L} \mathcal{D} \xi_{R} \ldots e^{-W_{q}\left(\xi_{L}, \xi_{R}\right)}}{\int \mathcal{D} \xi_{L} \mathcal{D} \xi_{R} e^{-W_{q}\left(\xi_{L}, \xi_{R}\right)}} .
\end{aligned}
$$

It is worth noting that, as well as for the weak-coupling case, there will be self-energies coming from the subleading terms in the series. A convenient way to get rid of them is to apply a normal ordering, in such a way that $a$ independent contributions coming from contractions between points on the same vertex are discarded. In the weak-coupling case, that amounts to the usual normal ordering, since the free propagator is independent of $a$. Here, on the contrary, the propagator for the auxiliary fields does depend on $a$. Thus, the normal ordering subtracts from it a contribution which is independent of $a$. More explicitly, when including tadpoles, they contribute

$$
\left\langle\xi_{L}\left(x_{\|}\right) \xi_{L}\left(x_{\|}\right)\right\rangle_{q}=\int \frac{d^{3} k_{\|}}{(2 \pi)^{3}} \frac{2\left|k_{\|}\right|}{e^{2\left|k_{\|}\right| a}-1}=\left\langle\xi_{R}\left(x_{\|}\right) \xi_{R}\left(x_{\|}\right)\right\rangle_{q} .
$$

The leading term is then $\Gamma_{q}$ which is identical to the Dirichlet result, and therefore the corresponding energy per unit area is given by

$$
\mathcal{E}_{q}=-\frac{\pi^{2}}{1440 a^{3}}
$$

Let us now consider the expansion of $\Gamma_{s}$, which contains the subleading terms. Note that, since now the Gaussian average depends on $a$, even the first-order terms in $W_{L, R}$ may produce nontrivial contributions to the interaction energy. Indeed, we see that

$$
\Gamma_{s}^{(1)}=\left\langle W_{L}\left(\xi_{L}\right)\right\rangle_{q}+\left\langle W_{R}\left(\xi_{R}\right)\right\rangle_{q}
$$

As an example, let us consider

$$
W_{R}\left(\xi_{R}\right)=\int_{x_{\|}} \frac{g_{R}}{4 !}\left[\xi_{R}\left(x_{\|}\right)\right]^{4}, \quad W_{L}\left(\xi_{L}\right)=0,
$$

namely, a nonlinear mirror at $x_{3}=a$ and a Dirichlet one at $x_{3}=0$. In this case, we obtain

$$
\Gamma_{s}^{(1)}=\frac{g_{R}}{8}\left(\int \frac{d^{3} k_{\|}}{(2 \pi)^{3}} \frac{2\left|k_{\|}\right|}{e^{2\left|k_{\|}\right| a}-1}\right)^{2}=\frac{\pi^{6} g_{R}}{460800 a^{8}} .
$$

The following orders in an expansion of $\Gamma_{s}$ will involve terms which may be interpreted as involving more than one loop. For example, one sees that at the second order the contribution may be written as

$$
\begin{aligned}
\Gamma_{s}^{(2)}= & -\frac{1}{2}\left\langle\left[ W_{L}\left(\xi_{L}\right)+W_{R}\left(\xi_{R}\right)-\left\langle W_{L}\left(\xi_{L}\right)\right\rangle_{q}\right.\right. \\
& \left.\left.-\left\langle W_{R}\left(\xi_{R}\right)\right\rangle_{q}\right]^{2}\right\rangle_{q},
\end{aligned}
$$

which is negative definite.

\section{CONCLUSIONS}

We have presented a perturbative treatment for the calculation of the Casimir energy in a system where the boundary conditions, imposed on a real scalar field, are nonlinear. The expression for the Casimir interaction energy is expressed in terms of the constants that determine the boundary conditions, as well as the distance. In the nonlinear case, the mirrors' properties cannot be completely specified by just reflection and transmission coefficients, as it happens in the usual Lifshitz formula [19]. Our study has been concentrated on the case of a single real scalar field, as a first step towards understanding the more realistic, gauge field case. In many cases, the study of the Casimir effect for the electromagnetic field may be related to two real scalar fields, one of them with Dirichlet and the other with Neumann conditions (for perfectly conducting mirrors). We believe our study may be a first step towards the inclusion of nonlinear effects for the would-be scalar field which satisfies nonlinear Dirichlet-like conditions. The study of Neumann-like conditions could require the inclusion of derivatives of the field in the interaction terms.

We have considered different situations: first the case of semitransparent nonlinear mirrors, then mirrors including both linear and nonlinear contributions in their boundary conditions, and finally situations where the mirrors can be described as having a small nonlinear contribution on top of an otherwise perfect (Dirichlet) plane.

The nonlinearities manifest themselves, at this level, in the presence of terms in the interaction energy which have a nonstandard dependence on the distance $a$, even when they are semitransparent.

\section{ACKNOWLEDGMENTS}

C. D.F has been supported by CONICET, ANPCyT, and UNCuyo, Argentina. The Conselho Nacional de Desenvolvimento Científico e Tecnológico (CNPqBrazil), the Proppi-UFF, Capes, and FAPERJ are also acknowledged for financial support. 
[1] G. Plunien, B. Müller, and W. Greiner, Phys. Rep. 134, 87 (1986); P. Milonni, The Quantum Vacuum (Academic Press, San Diego, 1994); V. M. Mostepanenko and N. N. Trunov, The Casimir Effect and its Applications (Clarendon, London, 1997); M. Bordag, The Casimir Effect 50 Years Later (World Scientific, Singapore, 1999); M. Bordag, U. Mohideen, and V. M. Mostepanenko, Phys. Rep. 353, 1 (2001); K. A. Milton, The Casimir Effect: Physical Manifestations of the Zero-Point Energy (World Scientific, Singapore, 2001); S. Reynaud et al., C. R. Acad. Sci. Paris IV-2, 1287 (2001); K. A. Milton, J. Phys. A 37, R209 (2004); S. K. Lamoreaux, Rep. Prog. Phys. 68, 201 (2005); New J. Phys. 8, E05 (2006).

[2] E. Elizalde and S. D. Odintsov, Classical Quantum Gravity 12, 2881 (1995).

[3] A. Saharian and G. Esposito, J. Phys. A 39, 5233 (2006); in Proceedings of 11th Marcel Grossmann Meeting on General Relativity, Berlin, Germany, 23-29 Jul 2006 (World Scientific, Singapore, 2008), p. 2761.

[4] C. D. Fosco and E. Losada, Phys. Lett. B 675, 252 (2009).

[5] R. Esquivel-Sirvent, C. Villarreal, W. L. Mochán, A. M. Contreras-Reyes, and V. B. Svetovoy, J. Phys. A 39, 6323 (2006); R. Esquivel, C. Villarreal, and W. L. Mochán, Phys. Rev. A 68, 052103 (2003); R. Esquivel-Sirvent, C. Villarreal, and W. L. Mochán, Phys. Rev. A 71, 029904(E) (2005); R. Esquivel-Sirvent and W. L. Mochán, in Quantum Field Theory Under the Influence of External Conditions, edited by K. A. Milton and M. Bordag (World Scientific, Singapore, 2010).
[6] Z. Bajnok, L. Palla, and G. Takács, Phys. Rev. D 73, 065001 (2006); Nucl. Phys. B772, 290 (2007).

[7] See, for example, N. Graham, R. L. Jaffe, V. Khemani, M. Quandt, O. Schroeder, and H. Weigel, Nucl. Phys. B677, 379 (2004) and references therein.

[8] R. L. Jaffe, AIP Conf. Proc. 687, 3 (2003).

[9] C. D. Fosco, F. C. Lombardo, and F. D. Mazzitelli, Phys. Lett. B 669, 371 (2008).

[10] C. D. Fosco, F. C. Lombardo, and F. D. Mazzitelli, Phys. Rev. D 80, 085004 (2009).

[11] N. Bloembergen and P. S. Pershan, Phys. Rev. 128, 606 (1962).

[12] A. Ciattoni, C. Rizza, and E. Palange, Phys. Rev. A 81, 043839 (2010).

[13] M. Aparicio Alcalde, G. Flores Hidalgo, and N. F. Svaiter, J. Math. Phys. (N.Y.) 47, 052303 (2006).

[14] R. Moazzemi and S. S. Gousheh, Phys. Lett. B 658, 255 (2008).

[15] K. A. Milton, P. Parashar, and J. Wagner, Phys. Rev. Lett. 101, 160402 (2008).

[16] J. Wagner, K. A. Milton, and P. Parashar, J. Phys. Conf. Ser. 161, 012022 (2009).

[17] J. Zinn-Justin, Quantum field theory and critical phenomena (Clarendon, Oxford, 2002).

[18] J. I. Kapusta and C. Gale, Finite-Temperature Field Theory: Principles and Applications, 2nd ed. (Cambridge University Press, Cambridge, England, 2006).

[19] E. M. Lifshitz, Zh. Eksp. Teor. Fiz. 29, 94 (1955) [Sov. Phys. JETP 2, 73 (1956)]. 